\title{
A generating function method for the average-case analysis of DPLL
}

\author{
Rémi Monasson \\ CNRS-Laboratoire de Physique Théorique, ENS, Paris, France \\ monasson@lpt.ens.fr
}

\begin{abstract}
A method to calculate the average size of Davis-PutnamLoveland-Logemann (DPLL) search trees for random computational problems is introduced, and applied to the satisfiability of random CNF formulas (SAT) and the coloring of random graph (COL) problems. We establish recursion relations for the generating functions of the average numbers of (variable or color) assignments at a given height in the search tree, which allow us to derive the asymptotics of the expected DPLL tree size, $2^{N \omega+o(N)}$, where $N$ is the instance size. $\omega$ is calculated as a function of the input distribution parameters (ratio of clauses per variable for SAT, average vertex degree for COL), and the branching heuristics.
\end{abstract}

\section{Introduction and main results.}

Many efforts have been devoted to the study of the performances of the DavisPutnam-Loveland-Logemann (DPLL) procedure [19], and more generally, resolution proof complexity for combinatorial problems with randomly generated instances. Two examples are random $k$-Satisfiability ( $k$-SAT), where an instance $\mathcal{F}$ is a uniformly and randomly chosen set of $M=\alpha N$ disjunctions of $k$ literals built from $N$ Boolean variables and their negations (with no repetition and no complementary literals), and random graph $k$-Coloring ( $k$-COL), where an instance $\mathcal{F}$ is an Erdös-Rényi random graph from $G(N, p=c / N)$ i.e. with average vertex degree $c$.

Originally, efforts were concentrated on the random width distribution for $k$-SAT, where each literal appear with a fixed probability. Franco, Purdom and collaborators showed that simplified versions of DPLL had polynomial averagecase complexity in this case, see [24,14] for reviews. It was then recognized that the fixed clause length ensemble might provide harder instances for DPLL [12]. Chvátal and Szemerédi indeed showed that DPLL proof size is w.h.p. exponentially large (in $N$ at fixed ratio $\alpha$ ) for an unsatisfiable instance [9]. Later on, Beame et al. [4 showed that the proof size was w.h.p. bounded from above by 
$2^{c N / \alpha}$ (for some constant $c$ ), a decreasing function of $\alpha$. As for the satisfiable case, Frieze and Suen showed that backtracking is irrelevant at small enough ratios $\alpha$ ( $\leq 3.003$ with the Generalized Unit Clause heuristic, to be defined below) [15], allowing DPLL to find satisfying assignment in polynomial (linear) time. Achlioptas, Beame and Molloy proved that, conversely, at ratios smaller than the generally accepted satisfiability threshold, DPLL takes w.h.p. exponential time to find a satisfying assignment [3]. Altogether these results provide explanations for the 'easy-hard-easy' (or, more precisely, 'easy-hard-less hard') pattern of complexity experimentally observed when running DPLL on random 3-SAT instances [23].

A precise calculation of the average size of the search space explored by DPLL (and \#DPLL, a version of the procedure solving the enumeration problems \#SAT and \#COL) as a function of the parameters $N$ and $\alpha$ or $c$ is difficult due to the statistical correlations between branches in the search tree resulting from backtracking. Heuristic derivations were nevertheless proposed by Cocco and Monasson based on a 'dynamic annealing' assumption 10,11, 13. Hereafter, using the linearity of expectation, we show that 'dynamic annealing' turns not to be an assumption at all when the expected tree size is concerned.

We first illustrate the approach, based on the use of recurrence relations for the generating functions of the number of nodes at a given height in the tree, on the random $k$-SAT problem and the simple Unit Clause (UC) branching heuristic where unset variables are chosen uniformly at random and assigned to True or False uniformly at random [7,8]. Consider the following counting algorithm

Procedure \#DPLL-UC[F $\mathcal{F}, \mathrm{A}, \mathrm{S}]$

Call $\mathcal{F}_{A}$ what is left from instance $\mathcal{F}$ given partial variable assignment $A$;

1. If $\mathcal{F}_{A}$ is empty, $S \rightarrow S+2^{N-|A|}$, Return; (Solution Leaf)

2. If there is an empty clause in $\mathcal{F}_{A}$, Return; (Contradiction Leaf)

3. If there is no empty clause in $\mathcal{F}_{A}$, let $\Gamma_{1}=\left\{1\right.$-clauses $\left.\in \mathcal{F}_{A}\right\}$, if $\Gamma_{1} \neq \emptyset$, pick any 1-clause, say, $\ell$, and call DPLL $[\mathcal{F}, \mathrm{A} \cup \ell]$; (unit-propagation) if $\Gamma_{1}=\emptyset$, pick up an unset literal uniformly at random, say, $\ell$, and call $\operatorname{DPLL}[\mathcal{F}, \mathrm{A} \cup \ell]$, then DPLL $[\mathcal{F}, \mathrm{A} \cup \bar{\ell}] ;$ (variable splitting)

End;

\#DPLL-UC, called with $A=\emptyset$ and $S=0$, returns the number $S$ of solutions of the instance $\mathcal{F}$; the history of the search can be summarized as a search tree with leaves marked with solution or contradiction labels. As the instance to be 
treated and the sequence of operations done by \#DPLL-UC are stochastic, so are the numbers $L_{S}$ and $L_{C}$ of solution and contradiction leaves respectively.

Theorem 1. Let $k \geq 3$ and $\Omega(t, \alpha, k)=t+\alpha \log _{2}\left(1-\frac{k}{2^{k}} t^{k-1}+\frac{k-1}{2^{k}} t^{k}\right)$. The expectations of the numbers of solution and contradiction leaves in the \#DPLL$U C$ search tree of random $k$-SAT instances with $N$ variables and $\alpha N$ clauses are, respectively, $L_{S}(N, \alpha, k)=2^{N \omega_{S}(\alpha, k)+o(N)}$ with $\omega_{S}(\alpha, k)=\Omega(1, \alpha, k)$ and $L_{C}(N, \alpha, k)=2^{N \omega_{C}(\alpha, k)+o(N)}$ with $\omega_{C}(\alpha, k)=\max _{t \in[0 ; 1]} \Omega(t, \alpha, k)$.

An immediate consequence of Theorem 1 is that the expectation value of the total number of leaves, $L_{S}+L_{C}$, is $2^{N \omega_{C}(\alpha, k)+o(N)}$. This result was first found by Méjean, Morel and Reynaud in the particular case $k=3$ and for ratios $\alpha>1$ [22. Our approach not only provides a much shorter proof, but can also be easily extended to other problems and more sophisticated heuristics, see Theorems 2 and 3 below. In addition, Theorem 1 provides us with some information about the expected search tree size of the decision procedure DPLL-UC, corresponding to \#DPLL-UC with Line 1 replaced with: If $\mathcal{F}_{A}$ is empty, output Satisfiable; Halt.

Corollary 1. Let $\alpha>\alpha_{u}(k)$, the root of $\omega_{C}(\alpha, k)=2+\alpha \log _{2}\left(1-2^{-k}\right)$ e.g. $\alpha_{u}(3)=10.1286 \ldots$. The average size of DPLL-UC search trees for random $k-S A T$ instances with $N$ variables and $\alpha N$ clauses equals $2^{N \omega_{C}(\alpha, k)+o(N)}$.

Functions $\omega_{S}, \omega_{C}$ are shown in Figure 1 in the $k=3$ case. They coincide and are equal to $1-\alpha \log _{2}(8 / 7)$ for $\alpha<\alpha^{*}=4.56429 \ldots$, while $\omega_{C}>\omega_{S}$ for $\alpha>\alpha^{*}$. In other words, for $\alpha>\alpha^{*}$, most leaves in \#DPLL-UC trees are contradiction leaves, while for $\alpha<\alpha^{*}$, both contradiction and solution leaf numbers are (to exponential order in $N$ ) of the same order. As for DPLL-UC trees, notice that $\omega_{C}(\alpha, k) \asymp \frac{2 \ln 2}{3 \alpha}=\frac{0.46209 \ldots}{\alpha}$. This behaviour agrees with Beame et al.'s result $(\Theta(1 / \alpha))$ for the average resolution proof complexity of unsatisfiable instances [4]. Corollary 1 shows that the expected DPLL tree size can be estimated for a whole range of $\alpha$; we conjecture that the above expression holds for ratios smaller than $\alpha_{u}$ i.e. down to $\alpha^{*}$ roughly. For generic $k \geq 3$, we have $\omega_{C}(\alpha, k) \asymp \frac{k-2}{k-1}\left(\frac{2^{k} \ln 2}{k(k-1) \alpha}\right)^{1 /(k-2)}$; the decrease of $\omega_{C}$ with $\alpha$ is therefore slower and slower as $k$ increases.

So far, no expression for $\omega$ has been obtained for more sophisticated heuristics than UC. We consider the Generalized Unit Clause (GUC) heuristic [8,2] 


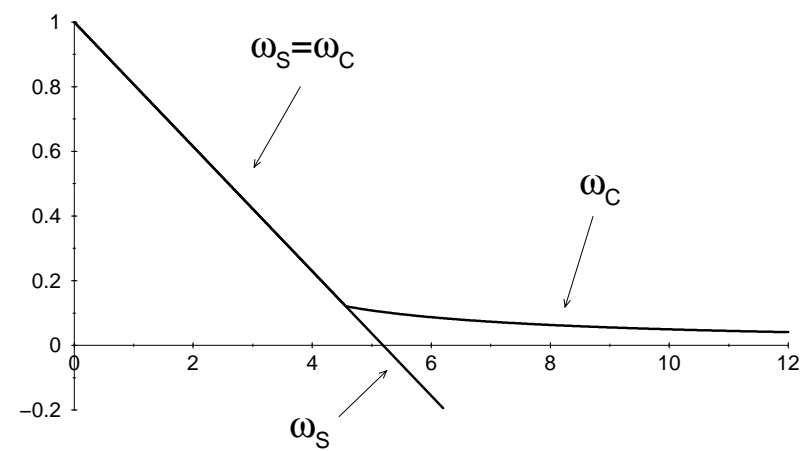

Fig. 1. Logarithms of the average numbers of solution and contradiction leaves, respectively $\omega_{S}$ and $\omega_{C}$, in \#DPLL-UC search trees versus ratio $\alpha$ of clauses per variable for random 3-SAT. Notice that $\omega_{C}$ coincides with the logarithm of the expected size of \#DPLL-UC at all ratios $\alpha$, and with the one of DPLL-UC search trees for $\alpha \geq 10.1286 \ldots$.

where the shortest clauses are preferentially satisfied. The associated decision procedure, DPLL-GUC, corresponds to DPLL-UC with Line 3 replaced with: Pick a clause uniformly at random among the shortest clauses, and a literal, say, $\ell$, in the clause; call DPLL $[\mathcal{F}, A \cup \ell]$, then $\operatorname{DPLL}[\mathcal{F}, A \cup \bar{\ell}]$.

Theorem 2. Define $m\left(x_{2}\right)=\frac{1}{2}\left(1+\sqrt{1+4 x_{2}}\right)-2 x_{2}, y_{3}\left(y_{2}\right)$ the solution of the ordinary differential equation $d y_{3} / d y_{2}=3\left(1+y_{2}-2 y_{3}\right) /\left(2 m\left(y_{2}\right)\right)$ such that $y_{3}(1)=1$, and

$\omega^{g}(\alpha)=\max _{\frac{3}{4}<y_{2} \leq 1}\left[\int_{y_{2}}^{1} \frac{d z}{m(z)} \log _{2}(2 z+m(z)) \exp \left(-\int_{z}^{1} \frac{d w}{m(w)}\right)+\alpha \log _{2} y_{3}\left(y_{2}\right)\right]$.

Let $\alpha>\alpha_{u}^{g}=10.2183 \ldots$, the root of $\omega^{g}(\alpha)+\alpha \log _{2}(8 / 7)=2$. The expected size of DPLL-GUC search tree for random 3-SAT instances with $N$ variables and $\alpha N$ clauses is $2^{N \omega^{g}(\alpha)+o(N)}$.

Notice that, at large $\alpha, \omega^{g}(\alpha) \asymp \frac{3+\sqrt{5}}{6 \ln 2}\left[\ln \left(\frac{1+\sqrt{5}}{2}\right)\right]^{2} \frac{1}{\alpha}=\frac{0.29154 \ldots}{\alpha}$ in agreement with the $1 / \alpha$ scaling established in [- 4 . Furthermore, the multiplicative factor is smaller than the one for UC, showing that DPLL-GUC is more efficient than DPLL-UC in proving unsatisfiability.

A third application is the analysis of the counterpart of GUC for the random 3-COL problem. The version of DPLL we have analyzed operates as follows [1]. 
Initially, each vertex is assigned a list of 3 available colors. In the course of the procedure, a vertex, say, $v$, with the smallest number of available colors, say, $j$, is chosen at random and uniformly. DPLL-GUC then removes $v$, and successively branches to the $j$ color assignments corresponding to removal of one of the $j$ colors of $v$ from the lists of the neighbors of $v$. The procedure backtracks when a vertex with no color left is created (contradiction), or no vertex is left (a proper coloring is found).

Theorem 3. Define $\omega^{h}(c)=\max _{0<t<1}\left[\frac{c}{6} t^{2}-\frac{c}{3} t-(1-t) \ln 2+\ln \left(3-e^{-2 c t / 3}\right)\right]$. Let $c>c_{u}^{h}=13.1538 \ldots$, the root of $\omega^{h}(c)+\frac{c}{6}=2 \ln 3$. The expected size of $D P L L-G U C$ search tree for deciding 3-COL on random graphs from $G(N, c / N)$ with $N$ vertices is $e^{N \omega^{h}(c)+o(N)}$.

Asymptotically, $\omega^{h}(c) \asymp \frac{3 \ln 2}{2 c^{2}}=\frac{1.0397 \ldots}{c^{2}}$ in agreement with Beame et al.'s scaling $\left(\Theta\left(1 / c^{2}\right)\right)$ 河. An extension of Theorem 3 to higher values of the number $k$ of colors gives $\omega^{h}(c, k) \asymp \frac{k(k-2)}{k-1}\left[\frac{2 \ln 2}{k-1}\right]^{1 /(k-2)} c^{-(k-1) /(k-2)}$. This result is compatible with the bounds derived in $\left[5\right.$, and suggests that the $\Theta\left(c^{-(k-1) /(k-2)}\right)$ dependence could hold w.h.p. (and not only in expectation).

\section{Recurrence equation for \#DPLL-UC search tree}

Let $\mathcal{F}$ be an instance of the 3-SAT problem defined over a set of $N$ Boolean variables $X$. A partial assignment $A$ of length $T(\leq N)$ is the specification of the truth values of $T$ variables in $X$. We denote by $\mathcal{F}_{A}$ the residual instance given $A$. A clause $c \in \mathcal{F}_{A}$ is said to be a $\ell$-clause with $\ell \in\{0,1,2,3\}$ if the number of false literals in $c$ is equal to $3-\ell$. We denote by $C_{\ell}\left(\mathcal{F}_{A}\right)$ the number of $\ell$-clauses in $\mathcal{F}_{A}$. The instance $\mathcal{F}$ is said to be satisfied under $A$ if $C_{\ell}\left(\mathcal{F}_{A}\right)=0$ for $\ell=$ $0,1,2,3$, unsatisfied (or violated) under $A$ if $C_{0}\left(\mathcal{F}_{A}\right) \geq 1$, undetermined under $A$ otherwise. The clause vector of an undetermined or satisfied residual instance $\mathcal{F}_{A}$ is the three-dimensional vector $\boldsymbol{C}$ with components $C_{1}\left(\mathcal{F}_{A}\right), C_{2}\left(\mathcal{F}_{A}\right), C_{3}\left(\mathcal{F}_{A}\right)$. The search tree associated to an instance $\mathcal{F}$ and a run of \#DPLL is the tree whose nodes carry the residual assignments $A$ considered in the course of the search. The height $T$ of a node is the length of the attached assignment.

It was shown by Chao and Franco [7,8] that, during the first descent in the search tree i.e. prior to any backtracking, the distribution of residual instances 
remains uniformly random conditioned on the numbers of $\ell$-clauses. This statement remains correct for heuristics more sophisticated than UC e.g. GUC, $\mathrm{SC}_{1}$ [8.2], and was recently extended to splitting heuristics based on variable occurrences by Kaporis, Kirousis and Lalas [18]. Clearly, in this context, uniformity is lost after backtracking enters into play (with the exception of Suen and Frieze's analysis of a limited version of backtracking [15]). Though this limitation appears to forbid (and has forbidden so far) the extension of average-case studies of backtrack-free DPLL to full DPLL with backtracking, we point out here that it is not as severe as it looks. Indeed, let us forget about how \#DPLL or DPLL search tree is built and consider its final state. We refer to a branch (of the search tree) as the shortest path from the root node (empty assignment) to a leaf. The two key remarks underlying the present work can be informally stated as follows. First, the expected size of a \#DPLL search tree can be calculated from the knolwedge of the statistical distribution of (residual instances on) a single branch; no characterization of the correlations between distinct branches in the tree is necessary. Secondly, the statistical distribution of (residual instances on) a single branch is simple since, along a branch, uniformity is preserved (as in the absence of backtracking). More precisely,

Lemma 1 (from Chao \& Franco [7]). Let $\mathcal{F}_{A}$ be a residual instance attached to a node $A$ at height $T$ in a \#DPLL-UC search tree produced from an instance $\mathcal{F}$ drawn from the random 3-SAT distribution. Then the set of $\ell$-clauses in $\mathcal{F}_{A}$ is uniformly random conditioned on its size $C_{\ell}\left(\mathcal{F}_{A}\right)$ and the number $N-T$ of unassigned variables for each $\ell \in\{0,1,2,3\}$.

Proof. the above Lemma is an immediate application of Lemma 3 in Achlioptas' Card Game framework which establishes uniformity for algorithms (a) 'pointing to a particular card (clause)', or (b) 'naming a variable that has not yet been assigned a value' (Section 2.1 in Ref. [2]). The operation of \#DPLL-UC along a branch precisely amounts to these two operations: unit-propagation relies on action $(a)$, and variable splitting on $(b)$.

Lemma 1 does not address the question of uniformity among different branches. Residual instances attached to two (or more) nodes on distinct branches in the search tree are correlated. However, these correlations can be safely ignored in calculating the average number of residual instances, in much the same way as 
the average value of the sum of correlated random variables is simply the sum of their average values.

Proposition 1. Let $L(\boldsymbol{C}, T)$ be the expectation of the number of undetermined residual instances with clause vector $\boldsymbol{C}$ at height $T$ in \#DPLL-UC search tree, and $G\left(x_{1}, x_{2}, x_{3} ; T\right)=\sum_{\boldsymbol{C}} x_{1}^{C_{1}} x_{2}^{C_{2}} x_{3}^{C_{3}} L(\boldsymbol{C}, T)$ its generating function. Then, for $0 \leq T<N$,

$$
\begin{aligned}
G\left(x_{1}, x_{2}, x_{3} ; T+1\right) & =\frac{1}{f_{1}} G\left(f_{1}, f_{2}, f_{3} ; T\right)+\left(2-\frac{1}{f_{1}}\right) G\left(0, f_{2}, f_{3} ; T\right) \\
& -2 G(0,0,0 ; T)
\end{aligned}
$$

where $f_{1}, f_{2}, f_{3}$ stand for the functions $f_{1}^{(T)}\left(x_{1}\right)=x_{1}+\frac{1}{2} \mu\left(1-2 x_{1}\right), f_{2}^{(T)}\left(x_{1}, x_{2}\right)=$ $x_{2}+\mu\left(x_{1}+1-2 x_{2}\right), f_{3}^{(T)}\left(x_{2}, x_{3}\right)=x_{3}+\frac{3}{2} \mu\left(x_{2}+1-2 x_{3}\right)$, and $\mu=1 /(N-T)$. The generating function $G$ is entirely defined from recurrence relation (2) and the initial condition $G\left(x_{1}, x_{2}, x_{3} ; 0\right)=\left(x_{3}\right)^{\alpha N}$.

Proof. Let $\delta_{n}$ denote the Kronecker function ( $\delta_{n}=1$ if $n=0, \delta_{n}=0$ otherwise), $B_{n}^{m, q}=\left(\begin{array}{c}m \\ n\end{array}\right) q^{n}(1-q)^{m-n}$ the binomial distribution. Let $A$ be a node at height $T$, and $\mathcal{F}_{A}$ the attached residual instance. Call $\boldsymbol{C}$ the clause vector of $\mathcal{F}_{A}$. Assume first that $C_{1} \geq 1$. Pick up one 1 -clause, say, $\ell$. Call $z_{j}$ the number of $j$-clauses that contain $\bar{\ell}$ or $\ell$ (for $j=1,2,3$ ). From Lemma 1 , the $z_{j}$ 's are binomial variables with parameter $j /(N-T)$ among $C_{j}-\delta_{j-1}$ (the 1-clause that is satisfied through unit-propagation is removed). Among the $z_{j}$ clauses, $w_{j-1}$ contained $\bar{\ell}$ and are reduced to $(j-1)$-clauses, while the remaining $z_{j}-w_{j-1}$ contained $\ell$ and are satisfied and removed. From Lemma 1 again, $w_{j-1}$ is a binomial variable with parameter $1 / 2$ among $z_{j}$. The probability that the instance produced has no empty clause $\left(w_{0}=0\right)$ is $B_{0}^{z_{1}, \frac{1}{2}}=2^{-z_{1}}$. Thus, setting $\mu=\frac{1}{N-T}$,

$$
\begin{aligned}
M_{P} & {\left[\boldsymbol{C}^{\prime}, \boldsymbol{C} ; T\right]=\sum_{z_{3}=0}^{C_{3}} B_{z_{3}}^{C_{3}, 3 \mu} \sum_{w_{2}=0}^{z_{3}} B_{w_{2}}^{z_{3}, \frac{1}{2}} \sum_{z_{2}=0}^{C_{2}} B_{z_{2}}^{C_{2}, 2 \mu} \sum_{w_{1}=0}^{z_{2}} B_{w_{1}}^{z_{2}, \frac{1}{2}} } \\
& \times \sum_{z_{1}=0}^{C_{1}-1} B_{z_{1}}^{C_{1}-1, \mu} \frac{1}{2^{z_{1}}} \delta_{C_{3}^{\prime}-\left(C_{3}-z_{3}\right)} \delta_{C_{2}^{\prime}-\left(C_{2}-z_{2}+w_{2}\right)} \delta_{C_{1}^{\prime}-\left(C_{1}-1-z_{1}+w_{1}\right)}
\end{aligned}
$$

expresses the probability that a residual instance at height $T$ with clause vector $\boldsymbol{C}$ gives rise to a (non-violated) residual instance with clause vector $\boldsymbol{C}^{\prime}$ at height $T+1$ through unit-propagation. Assume now $C_{1}=0$. Then, a yet unset variable 
is chosen and set to True or False uniformly at random. The calculation of the new vector $C^{\prime}$ is identical to the unit-propagation case above, except that: $z_{1}=w_{0}=0$ (absence of 1-clauses), and two nodes are produced (instead of one). Hence,

$$
\begin{aligned}
M_{U C}\left[\boldsymbol{C}^{\prime}, \boldsymbol{C} ; T\right]= & 2 \sum_{z_{3}=0}^{C_{3}} B_{z_{3}}^{C_{3}, 3 \mu} \sum_{w_{2}=0}^{z_{3}} B_{w_{2}}^{z_{3}, \frac{1}{2}} \sum_{z_{2}=0}^{C_{2}} B_{z_{2}}^{C_{2}, 2 \mu} \sum_{w_{1}=0}^{z_{2}} B_{w_{1}}^{z_{2}, \frac{1}{2}} \\
& \times \delta_{C_{3}^{\prime}-\left(C_{3}-z_{3}\right)} \delta_{C_{2}^{\prime}-\left(C_{2}-z_{2}+w_{2}\right)} \delta_{C_{1}^{\prime}-w_{1}}
\end{aligned}
$$

expresses the expected number of residual instances at height $T+1$ and with clause vector $C^{\prime}$ produced from a residual instance at height $T$ and with clause vector $C$ through $\mathrm{UC}$ branching.

Now, consider all the nodes $A_{i}$ at height $T$, with $i=1, \ldots, \mathcal{L}$. Let $o_{i}$ be the operation done by \#DPLL-UC on $A_{i} . o_{i}$ represents either unit-propagation (literal $\ell_{i}$ set to True) or variable splitting (literals $\ell_{i}$ set to $\mathrm{T}$ and $\mathrm{F}$ on the descendent nodes respectively). Denoting by $\mathbf{E}_{Y}(X)$ the expectation value of a quantity $X$ over variable $Y, L\left(\boldsymbol{C}^{\prime} ; T+1\right)=\mathbf{E}_{\mathcal{L},\left\{A_{i}, o_{i}\right\}}\left(\sum_{i=1}^{\mathcal{L}} \mathcal{M}\left[\boldsymbol{C}^{\prime} ; A_{i}, o_{i}\right]\right)$ where $\mathcal{M}$ is the number $(0,1$ or 2$)$ of residual instances with clause vector $\boldsymbol{C}^{\prime}$ produced from $A_{i}$ after \#DPLL-UC has carried out operation $o_{i}$. Using the linearity of expectation, $L\left(\boldsymbol{C}^{\prime} ; T+1\right)=\mathbf{E}_{\mathcal{L}}\left(\sum_{i=1}^{\mathcal{L}} \mathbf{E}_{\left\{A_{i}, o_{i}\right\}}\left(\mathcal{M}\left[\boldsymbol{C}^{\prime} ; A_{i}, o_{i}\right]\right)\right)=\mathbf{E}_{\mathcal{L}}\left(\sum_{i=1}^{\mathcal{L}} M\left[\boldsymbol{C}^{\prime}, \boldsymbol{C}_{i} ; T\right]\right)$ where $C_{i}$ is the clause vector of the residual instance attached to $A_{i}$, and $M\left[\boldsymbol{C}^{\prime}, \boldsymbol{C} ; T\right]=\left(1-\delta_{C_{1}}\right) M_{P}\left[\boldsymbol{C}^{\prime}, \boldsymbol{C} ; T\right]+\delta_{C_{1}} M_{U C}\left[\boldsymbol{C}^{\prime}, \boldsymbol{C} ; T\right]$. Gathering assignments with identical clause vectors gives the reccurence relation $L\left(\boldsymbol{C}^{\prime}, T+1\right)=$ $\sum_{\boldsymbol{C}} M\left[\boldsymbol{C}^{\prime}, \boldsymbol{C} ; T\right] L(\boldsymbol{C}, T)$. Recurrence relation (2) for the generating function is an immediate consequence. The initial condition over $G$ stems from the fact that the instance is originally drawn from the random 3-SAT distribution, $L(\boldsymbol{C} ; 0)=$ $\delta_{C_{1}} \delta_{C_{2}} \delta_{C_{3}-\alpha N}$.

\section{Asymptotic analysis and application to DPLL-UC}

The asymptotic analysis of $G$ relies on the following technical lemma:

Lemma 2. Let $\gamma\left(x_{2}, x_{3}, t\right)=(1-t)^{3} x_{3}+\frac{3 t}{2}(1-t)^{2} x_{2}+\frac{t}{8}\left(12-3 t-2 t^{2}\right)$, with $t \in] 0 ; 1\left[\right.$ and $x_{2}, x_{3}>0$. Define $S_{0}(T) \equiv \sum_{H=0}^{T} 2^{T-H} G(0,0,0 ; H)$. Then, in 
the large $N$ limit, $S_{0}([t N]) \leq 2^{N\left(t+\alpha \log _{2} \gamma(0,0, t)\right)+o(N)}$ and $G\left(\frac{1}{2}, x_{2}, x_{3} ;[t N]\right)=$ $2^{N\left(t+\alpha \log _{2} \gamma\left(x_{2}, x_{3}, t\right)\right)+o(N)}$.

Due to space limitations, we give here only some elements of the proof. The first step in the proof is inspired by Knuth's kernel method [20]: when $x_{1}=\frac{1}{2}$, $f_{1}=\frac{1}{2}$ and recurrence relation (2) simplifies and is easier to handle. Iterating this equation then allows us to relate the value of $G$ at height $T$ and coordinates $\left(\frac{1}{2}, x_{2}, x_{3}\right)$ to the (known) value of $G$ at height 0 and coordinates $\left(\frac{1}{2}, y_{2}, y_{3}\right)$ which are functions of $x_{2}, x_{3}, T, N$, and $\alpha$. The function $\gamma$ is the value of $y_{3}$ when $T, N$ are sent to infinity at fixed ratio $t$. The asymptotic statement about $S_{0}(T)$ comes from the previous result and the fact that the dominant terms in the sum defining $S_{0}$ are the ones with $H$ close to $T$.

Proposition 2. Let $L_{C}(N, T, \alpha)$ be the expected number of contradiction leaves of height $T$ in the \#DPLL-UC resolution tree of random 3-SAT instances with $N$ variables and $\alpha N$ clauses, and $\epsilon>0$. Then, for $t \in[\epsilon ; 1-\epsilon]$ and $\alpha>0$, $\Omega(t, \alpha, 3) \leq \frac{1}{N} \log _{2} L_{C}(N,[t N], \alpha)+o(1) \leq \max _{h \in[\epsilon, ; t]} \Omega(h, \alpha, 3)$ where $\Omega$ is defined in Theorem 1.

Observe that a contradiction may appear with a positive (and non-exponentially small in $N$ ) probability as soon as two 1-clauses are present. These 1-clauses will be present as a result of 2-clause reduction when the residual instances include a large number $(\Theta(N))$ of 2-clauses. As this is the case for a finite fraction of residual instances, $G(1,1,1 ; T)$ is not exponentially larger than $L_{C}(T)$. Use of the monotonicity of $G$ with respect to $x_{1}$ and Lemma 2 gives the announced lower bound (recognize that $\Omega(t, \alpha, 3)=t+\alpha \log _{2} \gamma(1,1 ; t)$ ). To derive the upper bound, remark that contradictions leaves cannot be more numerous than the number of branches created through splittings; hence $L_{C}(T)$ is bounded from above by the number of splittings at smaller heights $H$, that is, $\sum_{H<T} G(0,1,1 ; H)$. Once more, we use the monotonicity of $G$ with respect to $x_{1}$ and Lemma 2 to obtain the upper bound. The complete proof will be given in the full version.

Proof. (Theorem 1) By definition, a solution leaf is a node in the search tree where no clauses are left; the average number $L_{S}$ of solution leaves is thus given by $L_{S}=\sum_{H=0}^{N} L(0,0,0 ; H)=\sum_{H=0}^{N} G(\mathbf{0} ; H)$. A straightforward albeit useful upper 
bound on $L_{S}$ is obtained from $L_{S} \leq S_{0}(N)$. By definition of the algorithm \#DPLL, $S_{0}(N)$ is the average number of solutions of an instance with $\alpha N$ clauses over $N$ variables drawn from the random 3-SAT distribution, $S_{0}(N)=$ $2^{N}(7 / 8)^{\alpha N}$ [12]. This upper bound is indeed tight (to within terms that are subexponential in $N$ ), as most solution leaves have heights equal, or close to $N$. To show this, consider $\epsilon>0$, and write

$L_{S} \geq \sum_{H=N(1-\epsilon)}^{N} G(\mathbf{0} ; H) \geq 2^{-N \epsilon} \sum_{H=N(1-\epsilon)}^{N} 2^{N-H} G(\mathbf{0} ; H)=2^{-N \epsilon} S_{0}(N)[1-A]$ with $A=2^{N \epsilon} S_{0}(N(1-\epsilon)) / S_{0}(N)$. From Lemma $2, A \leq(\kappa+o(1))^{\alpha N}$ with $\kappa=\frac{\gamma(0,0,1-\epsilon)}{7 / 8}=1-\frac{9}{7} \epsilon^{2}+\frac{2}{7} \epsilon^{3}<1$ for small enough $\epsilon$ (but $\Theta(1)$ with respect to $N)$. We conclude that $A$ is exponential small in $N$, and $-\epsilon+1-$ $\alpha \log _{2} \frac{8}{7}+o(1) \leq \frac{1}{N} \log _{2} L_{S} \leq 1-\alpha \log _{2} \frac{8}{7}$. Choosing arbitrarily small $\epsilon$ allows us to establish the statement about the asymptotic behaviour of $L_{S}$ in Theorem 1 .

Proposition 2, with arbitrarily small $\epsilon$, immediately leads to Theorem 1 for $k=3$, for the average number of contradiction leaves, $L_{C}$, equals the sum over all heights $T=t N$ (with $0 \leq t \leq 1$ ) of $L_{C}(N, T, \alpha)$, and the sum is bounded from below by its largest term and, from above, by $N$ times this largest term. The statement on the number of leaves following Theorem 1 comes from the observation that the expected total number of leaves is $L_{S}+L_{C}$, and $\omega_{S}(\alpha, 3)=\Omega(1, \alpha, 3) \leq \max _{t \in[0 ; 1]} \Omega(t, \alpha, 3)=\omega_{C}(\alpha, 3)$.

Proof. (Corollary 1) Let $P_{\text {sat }}$ be the probability that a random 3-SAT instance with $N$ variables and $\alpha N$ clauses is satisfiable. Define $\# L_{\text {sat }}$ and $\# L_{\text {unsat }}$ (respectively, $L_{\text {sat }}$ and $L_{\text {unsat }}$ ) the expected numbers of leaves in \#DPLL-UC (resp. DPLL-UC) search trees for satisfiable and unsatisfiable instances respectively. All these quantities depend on $\alpha$ and $N$. As the operations of \#DPLL and DPLL coincide for unsatifiable instances, we have $\# L_{\text {unsat }}=L_{\text {unsat }}$. Conversely, $\# L_{\text {sat }} \geq L_{\text {sat }}$ since DPLL halts after having encountered the first solution leaf. Therefore, the difference between the average sizes \#L and L of \#DPLL-UC and DPLL-UC search trees satisfies $0 \leq \# L-L=P_{\text {sat }}\left(\# L_{\text {sat }}-L_{\text {sat }}\right) \leq P_{\text {sat }} \# L_{\text {sat }}$. Hence, $1-P_{\text {sat }} \# L_{\text {sat }} / \# L \leq L / \# L \leq 1$. Using $\# L_{\text {sat }} \leq 2^{N}, P_{\text {sat }} \leq 2^{N}(7 / 8)^{\alpha N}$ from the first moment theorem and the asymptotic scaling for $\# L$ given in Theorem 1 , we see that the left hand side of the previous inequality tends to 1 when $N \rightarrow \infty$ and $\alpha>\alpha_{u}$. 
Proofs for higher values of $k$ are identical, and will be given in the full version.

\section{The GUC heuristic for random SAT and COL}

The above analysis of the DPLL-UC search tree can be extended to the GUC heuristic [8], where literals are preferentially chosen to satisfy 2-clauses (if any). The outlines of the proofs of Theorems 2 and 3 are given below; details will be found in the full version.

3-SAT. The main difference with respect to the UC case is that the two branches issued from the split are not statistically identical. In fact, the literal $\ell$ chosen by GUC satisfies at least one clause, while this clause is reduced to a shorter clause when $\ell$ is set to False. The cases $C_{2} \geq 1$ and $C_{2}=0$ have also to be considered separately. With $f_{1}, f_{2}, f_{3}$ defined in the same way as in the UC case, we obtain

$$
\begin{aligned}
G\left(x_{1}, x_{2}, x_{3}\right. & ; T+1)=\frac{1}{f_{1}} G\left(f_{1}, f_{2}, f_{3} ; T\right)+\left(\frac{1+f_{1}}{f_{2}}-\frac{1}{f_{1}}\right) G\left(0, f_{2}, f_{3} ; T\right) \\
& +\left(\frac{1+f_{2}}{f_{3}}-\frac{1+f_{1}}{f_{2}}\right) G\left(0,0, f_{3} ; T\right)-\frac{1+f_{2}}{f_{3}} G(0,0,0 ; T) .
\end{aligned}
$$

The asymptotic analysis of $G$ follows the lines of Section 3. Choosing $f_{2}=f_{1}+f_{1}^{2}$ i.e. $x_{1}=\left(-1+\sqrt{1+4 x_{2}}\right) / 2+O(1 / N)$ allows us to cancel the second term on the r.h.s. of (3). Iterating relation (3), we establish the counterpart of Lemma 2 for GUC: the value of $G$ at height $[t N]$ and argument $x_{2}, x_{3}$ is equal to its (known) value at height 0 and argument $y_{2}, y_{3}$ times the product of factors $\frac{1}{f_{1}}$, up to an additive term, $A$, including iterates of the third and fourth terms on the right hand side of (3). $y_{2}, y_{3}$ are the values at 'time' $\tau=0$ of the solutions of the ordinary differential equations (ODE) $d Y_{2} / d \tau=-2 m\left(Y_{2}\right) /(1-\tau), d Y_{3} / d \tau=$ $-3\left(\left(1+Y_{2}\right) / 2-Y_{3}\right) /(1-\tau)$ with 'initial' condition $Y_{2}(t)=x_{2}, Y_{3}(t)=x_{3}$ (recall that function $m$ is defined in Theorem 2). Eliminating 'time' between $Y_{2}, Y_{3}$ leads to the ODE in Theorem 2. The first term on the r.h.s. in the expression of $\omega^{g}$ (值) corresponds to the logarithm of the product of factors $\frac{1}{f_{1}}$ between heights 0 and $T$. The maximum over $y_{2}$ in expression (1) for $\omega^{g}$ is equivalent to the maximum over the reduced height $t$ appearing in $\omega_{C}$ in Theorem 1 (see also Proposition 2). Finally, choosing $\alpha>\alpha_{u}^{g}$ ensures that, from the one hand, the additive term $A$ mentioned above is asymptotically negligible and, from the other hand, the ratio 
of the expected sizes of \#DPLL-GUC and DPLL-GUC is asymptotically equal to unity (see proof of Corollary 1 ).

3-COL. The uniformity expressed by Lemma 1 holds: the subgraph resulting from the coloring of $T$ vertices is still Erdős-Rényi-like with edge probability $\frac{c}{N}$, conditioned to the numbers $C_{j}$ of vertices with $j$ available colors [1]. The generating function $G$ of the average number of residual asignments equals $\left(x_{3}\right)^{N}$ at height $T=0$ and obeys the reccurence relation, for $T<N$,

$$
\begin{aligned}
G\left(x_{1}, x_{2}, x_{3} ; T+1\right) & =\frac{1}{f_{1}} G\left(f_{1}, f_{2}, f_{3} ; T\right)+\left(\frac{2}{f_{2}}-\frac{1}{f_{1}}\right) G\left(0, f_{2}, f_{3} ; T\right) \\
& +\left(\frac{3}{f_{3}}-\frac{2}{f_{2}}\right) G\left(0,0, f_{3} ; T\right)
\end{aligned}
$$

with $f_{1}=(1-\mu) x_{1}, f_{2}=(1-2 \mu) x_{2}+2 \mu x_{1}, f_{3}=(1-3 \mu) x_{3}+3 \mu x_{2}$, and $\mu=c /(3 N)$. Choosing $f_{1}=\frac{1}{2} f_{2}$ i.e. $x_{1}=\frac{1}{2} x_{2}+O(1 / N)$ allows us to cancel the second term on the r.h.s. of (何). Iterating relation (3), we establish the counterpart of Lemma 2 for GUC: the value of $G$ at height $[t N]$ and argument $x_{2}, x_{3}$ is equal to its (known) value at height 0 and argument $y_{2}, y_{3}$ respectively, times the product of factors $\frac{1}{f_{1}}$, up to an additive term, $A$, including iterates of the last term in (14). An explicit calculation leads to $G\left(\frac{1}{2} x_{2}, x_{2}, x_{3} ;[t N]\right)=$ $e^{N \gamma^{h}\left(x_{2}, x_{3}, t\right)+o(N)}+A$ for $x_{2}, x_{3}>0$, where $\gamma^{h}\left(x_{2}, x_{3}, t\right)=\frac{c}{6} t^{2}-\frac{c}{3} t+(1-$ t) $\ln \left(x_{2} / 2\right)+\ln \left[3+e^{-2 c t / 3}\left(2 x_{2} / x_{3}-3\right)\right]$. As in Proposition 2, we bound from below (respectively, above) the number of contradiction leaves in \#DPLL-GUC tree by the exponential of ( $N$ times) the value of function $\gamma^{h}$ in $x_{2}=x_{3}=1$ at reduced height $t$ (respectively, lower than $t$ ). The maximum over $t$ in Theorem 3 is equivalent to the maximum over the reduced height $t$ appearing in $\omega_{C}$ in Theorem 1 (see also Proposition 2). Finally, we choose $c_{u}^{h}$ to make the additive term $A$ negligible. Following the notations of Corollary 1 , we use $L_{\text {sat }} \leq 3^{N}$, and $P_{\text {sat }} \leq 3^{N} e^{-N c / 6+o(N)}$, the expected number of 3-colorings for random graphs from $G(N, c / N)$.

\section{Conclusion and perspectives}

We emphasize that the average \#DPLL tree size can be calculated for even more complex heuristics e.g. making decisions based on literal degrees [18]. This task requires, in practice, that one is able: first, to find the correct conditioning ensuring uniformity along a branch (as in the study of DPLL in the absence of 
backtracking); secondly, to determine the asymptotic behaviour of the associated generating function $G$ from the recurrence relation for $G$.

To some extent, the present work is an analytical implementation of an idea put forward by Knuth thirty years ago [21, 11]. Knuth indeed proposed to estimate the average computational effort required by a backtracking procedure through successive runs of the non-backtracking counterpart, each weighted in an appropriate way 21]. This weight is, in the language of Section II.B, simply the probability of a branch (given the heuristic under consideration) in \#DPLL search tree times $2^{S}$ where $S$ is the number of splits [1].

Since the amount of backtracking seems to have a heavy tail 16, 17, the expectation is often not a good predictor in practice. Knowledge of the second moment of the search tree size would be very precious; its calculation, currently under way, requires us to treat the correlations between nodes attached to distinct branches. Calculating the second moment is a step towards the distant goal of finding the expectation of the logarithm, which probably requires a deep understanding of correlations as in the replica theory of statistical mechanics.

Last of all, \#DPLL is a complete procedure for enumeration. Understanding its average-case operation will, hopefully, provide us with valuable information not only on the algorithm itself but also on random decision problems e.g. new bounds on the sat/unsat or col/uncol thresholds, or insights on the statistical properties of solutions.

Acknowledgments: The present analysis is the outcome of a work started four years ago with S. Cocco to which I am deeply indebted 10,11. I am grateful to C. Moore for numerous and illuminating discussions, as well as for a critical reading of the manuscript. I thank J. Franco for his interest and support, and the referee for pointing out Ref. [6], the results of which agree with the $\alpha^{-1 /(k-2)}$ asymptotic scaling of $\omega$ found here.

\section{References}

1. Achlioptas, D. and Molloy, M. Analysis of a List-Coloring Algorithm on a Random Graph, in Proc. Foundations of Computer Science (FOCS), vol 97, 204 (1997).

2. Achlioptas, D. Lower bounds for random 3-SAT via differential equations, Theor. Comp. Sci. 265, 159-185 (2001).

3. Achlioptas, D., Beame, P. and Molloy, M. A Sharp Threshold in Proof Complexity. in Proceedings of STOC 01, p.337-346 (2001). 
4. Beame, P., Karp, R., Pitassi, T. and Saks, M. On the complexity of unsatisfiability of random $k$-CNF formulas. In Proceedings of the Thirtieth Annual ACM Symposium on Theory of Computing (STOC98), p. 561-571, Dallas, TX (1998).

5. Beame, P., Culberson, J., Mitchell, D. and Moore, C. The resolution complexity of random graph k-colorability. To appear in Discrete Applied Mathematics (2003).

6. Ben-Sasson, E. and Galesi, N. Space Complexity of Random Formulae in Resolution, Random Struct. Algorithms 23, 92 (2003).

7. Chao, M.T. and Franco, J. Probabilistic analysis of two heuristics for the 3satisfiability problem, SIAM Journal on Computing 15, 1106-1118 (1986).

8. Chao, M.T. and Franco, J. Probabilistic analysis of a generalization of the unit clause literal selection heuristics for the k-satisfiability problem, Information Science 51, 289-314 (1990).

9. Chvátal, V. and Szemerédi, E. Many hard examples for resolution, J. Assoc. Comput. Mach. 35, 759-768 (1988).

10. Cocco, S. and Monasson, R. Analysis of the computational complexity of solving random satisfiability problems using branch and bound search algorithms, Eur. Phys. J. B 22, 505 (2001).

11. Cocco, S. and Monasson, R. Heuristic average-case analysis of the backtrack resolution of random 3-Satisfiability instances. Theor. Comp. Sci. 320, 345 (2004).

12. Franco, J. and Paull, M. Probabilistic analysis of the Davis-Putnam procedure for solving the satisfiability problem. Discrete Appl. Math. 5, 77-87 (1983).

13. Ein-Dor, L. and Monasson, R. The dynamics of proving uncolourability of large random graphs: I. Symmetric colouring heuristic. J. Phys. A 36, 11055-11067 (2003).

14. Franco, J. Results related to thresholds phenomena research in satisfiability: lower bounds. Theor. Comp. Sci. 265, 147-157 (2001).

15. Frieze, A. and Suen, S. Analysis of two simple heuristics on a random instance of k-SAT. J. Algorithms 20, 312-335 (1996).

16. Gent, I. and Walsh, T. Easy problems are sometimes hard. Artificial Intelligence 70, 335 (1993).

17. Jia, H. and Moore, C. How much backtracking does it take to color random graphs? Rigorous results on heavy tails. In Proc. 10th International Conference on Principles and Practice of Constraint Programming (CP '04) (2004).

18. Kaporis, A.C., Kirousis, L.M. and Lalas, E.G. The Probabilistic Analysis of a Greedy Satisfiability Algorithm. ESA, p. 574-585 (2002).

19. Karp, R.M. The probabilistic analysis of some combinatorial search algorithms, in J.F. Traub, ed. Algorithms and Complexity, Academic Press, New York (1976).

20. Knuth, D.E. The art of computer programming, vol. 1: Fundamental algorithms, Section 2.2.1, Addison-Wesley, New York (1968).

21. Knuth, D.E. Estimating the efficiency of backtrack programs. Math. Comp. 29, $136(1975)$.

22. Méjean, H-M., Morel, H. and Reynaud, G. A variational method for analysing unit clause search. SIAM J. Comput. 24, 621 (1995).

23. Mitchell, D., Selman, B. and Levesque, H. Hard and Easy Distributions of SAT Problems, Proc. of the Tenth Natl. Conf. on Artificial Intelligence (AAAI-92), 440446, The AAAI Press / MIT Press, Cambridge, MA (1992).

24. Purdom, P.W. A survey of average time analyses of satisfiability algorithms. $J$. Inform. Process. 13, 449 (1990). 